\title{
Allelic diversity of the GJB2 gene in deaf patients and ethnically matched populations from South Siberia
}

\author{
M. Zytsar ${ }^{1,2 *}$, M. Bady-Khoo ${ }^{3}$, E. Maslova ${ }^{1,2}$, V. Danilchenko ${ }^{1,2}$, N. Barashkov ${ }^{4,5}$, \\ I. Morozov ${ }^{2,6}$, A. Bondar ${ }^{6}$, O. Posukh ${ }^{1,2}$ \\ ${ }^{1}$ Institute of Cytology and Genetics SB RAS, Novosibirsk, Russia \\ ${ }^{2}$ Novosibirsk State University, Novosibirsk, Russia \\ ${ }^{3}$ Scientific Research Institute of Medical-Social Problems and Management of the Republic of Tuva, \\ Kyzyl, Russia \\ ${ }^{4}$ Laboratory of Molecular Biology, MK Ammosov North-Eastern Federal University, Yakutsk, Russia \\ ${ }^{5}$ Laboratory of Molecular Genetics, Yakut Scientific Centre of Complex Medical Problems, Yakutsk, Russia \\ ${ }^{6}$ Institute of Chemical Biology and Fundamental Medicine SB RAS, Novosibirsk, Russia \\ *e-mail: zytzar@bionet.nsc.ru
}

Key words: hereditary deafness, GJB2, allelic diversity, populations of Siberia

Motivation and Aim: Pathogenic variants in gene GJB2 (MIM 121011, 13q11-q12) account for a significant portion of hereditary hearing loss (HL). Spectrum of GJB2 variations (pathogenic, benign and yet unclassified) and their prevalence are highly population-specific. We aimed to analyze allelic diversity of GJB2, obviously being under selection, in deaf patients from indigenous Siberian peoples (Tuvinians and Altaians), from Russian patients living in Siberia and in ethnically matched controls and to compare these data with appropriate data for worldwide populations.

Methods: Sanger sequencing was applied for analysis of non-coding (exon 1), coding (exon 2) and flanking intronic regions of the gene GJB2. Pedigree analysis and molecular cloning were applied for verification of cis-configuration of variants p.V27I and p.E114G. Genetic differentiation of populations was analyzed by standard population structure analysis softwares.

Results: Different spectrum of the GJB2 pathogenic variants (c. $-23+1 \mathrm{G}>\mathrm{A}, \mathrm{c} .35 \mathrm{delG}$, p.V37I, p.R75Q, c.235delC, c.299_300delAT, c.313_326del14, p.W172C) and their variable contribution to HL of patients were previously found $(15.1 \%$ and $17.5 \%$ in Altaians and Tuvinians, respectively, vs $55.9 \%$ in Russians). We also evaluated the frequencies of known and novel benign GJB2 variants: rs117685390, rs9552101, NC_000013.11:g.20193014C $>$ T, c.-23+27G $>$ A (rs899667206), p.V27I (rs2274084), p.E114G (rs2274083), p.V153I (rs111033186), p.F191L (rs397516878), p.I203T (rs76838169), rs3751385, rs5030700 in all examined samples. Ambiguous association of combination of variants p.V27I and p.E114G with HL is widely discussed in literature. Our important results: the proved cis-configuration of p.V27I and p.E114G and the absence of association ofallelep.[V27I;E114G] withHLbased on higher frequency ofp.[V27I;E114G] in Tuvinian and Altaian controls compared with the patient's samples. All obtained data were used for comparative analysis of genetic differentiation of studied samples and populations from global human genomic data (1000 Genomes, HapMap Projects etc). Conclusion: Despite the limited number of SNPs found in analyzed the GJB2 gene region, strong ethno-specific genetic differentiation was revealed between the samples of deaf patients and controls from indigenous Siberian populations vs corresponding samples of Russians.

Acknowledgements: Supported by the project 0324-2018-0016 and by the RFBR (18-34-00166_mol-a, 17-29-06016_ofi-m). 